\section{Zahlen des Monats}

\section{Schmerzen im Kreuz}

Rückenschmerzen sind oft ein Thema. Wie verbreitet das Volksleiden ist, zeigt der BARMER GEK Report Krankenhaus 2015. Danach ist die Zahl der Krankenhausfälle von Kreuzschmerzpatienten in den Jahren 2006 bis 2014 von 282.000 auf 415.000 angestiegen. Bei gut einem Drittel findet weder eine Operation an der Wirbelsäule noch eine spezifische Schmerztherapie statt. Laut Report gibt es bei rund 140.000 Betroffenen nur die bildgebende Diagnostik. Dagegen haben die OPs an den Bandscheiben um 12,2 Prozent zugenommen. Auch erhalten immer mehr Klinikpatienten eine Spritzen-Schmerztherapie. Die Leistung hat sich seit 2006 mehr als verdoppelt.

\section{Plus in der Kasse}

Die Apothekerkassen klingeln. Mit einem Umsatz von erstmals über 15 Milliarden Euro lag der Apothekenmarkt im ersten Halbjahr 2015 um 3,4 Prozent über dem im Vorjahreszeitraum. Das geht aus den Marktzahlen des Informationsdienstes INSIGHT Health hervor. Danach ist der Anteil der verschreibungspflichtigen Arzneimittel weiter gewachsen und binnen fünf Jahren von 81,9 auf jetzt 83,1 Prozent gestiegen. Den Umsatz in die Höhe treibt seit Jahren der kardiovaskuläre Bereich. Allerdings ist hier der generikafähige Markt gestiegen und hat die Umsätze gedrückt. Im Gegensatz dazu hat sich der Umsatz systemischer Antiinfektiva fast verdoppelt.

\section{Psycho-Stress in der Uni}

Spaß mit Kommilitonen, mehr Freizeit und ausgelassene WG-Partys: Viele Akademiker erinnern sich an ein tolles Studentenleben. Doch ist das Studium heute auch noch süß? Die Techniker Krankenkasse hat alarmierende Zahlen veröffentlicht. Danach erhielten im Jahr 2013 rund 21 Prozent der Studierenden eine psychische Diagnose. Frauen sind deutlich mehr betroffen. $\mathrm{Zu}$ den häufigsten Ursachen gehören Depressionen, somatoforme Störungen, Anpassungs-, Belastungs- sowie Angststörungen. Fast vier Prozent der Hochschüler bekamen 2014 Antidepressiva verordnet, das waren nach TKAngaben 43 Prozent mehr als 2006. $m f$

Regierung: Derzeit kein Handlungsbedarf \title{
GOZ bleibt unverändert
}

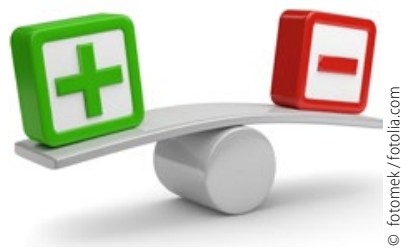

Wie der FVDZ aus gut unterrichteten Regierungskreisen erfahren hat, sieht die Bundesregierung bei der GOZ derzeit keinen Handlungsbedarf. Dies geht aus einem Bericht hervor, der am 19. August im Kabinett vorgelegt werden sollte. Darin heißt es unter anderem: Anhand der dargestellten Auswirkungen der GOZNovelle 2012 ergibt sich derzeit kein dringender Handlungsbedarf für eine Änderung der GOZ. Die weitere Entwicklung ist jedoch sorgfältig über einen längeren Zeitraum zu erfassen und zu analysieren. Demnach plant das Bundesgesundheitsministerium diese Analyse, nach Anpassung der Datengrundlage, im Jahr 2017 durchzuführen.

Dazu erklärte die FVDZ-Bundesvorsitzende Kerstin Blaschke: „Zynisch betrachtet könnten wir Zahnärzte jetzt der Bundesregierung dankbar sein, dass die GOZ 2012 unangetastet bleibt, und es keine Einschränkungen in finanzieller Hinsicht in den kommenden zwei Jahren geben wird. Wie gesagt: zynisch betrachtet. Aber in Wahrheit verweigert die Bundesregierung wieder einmal den Zahnärzten eine dringend notwendige Erhöhung der Gebühren. Es kann nicht sein, dass die GOZ zu einer Erstattungsordnung mutiert und sich an der finanziellen
Situation der Kostenträger orientiert. Ein Punktwert, der seit 1988 (!) bei 5,6241 Cent stagniert, hat die Bezeichnung „angemessen" in keiner Weise verdient. Das trägt weder den gestiegenen Kosten für Praxis noch für Personal oder Material Rechnung - von der seit 1988 aufgelaufenen Inflationsrate von 75 Prozent ganz zu schweigen. Selbst die Politik erkennt an dieser Stelle im Kabinettsentwurf, dass der Honoraranstieg von rund 9,2 Prozent den prognostizierten Honoraranstieg von sechs Prozent überschreitet. Die Begründung dafür lautet: Der Anteil der Material- und Laborkosten musste aufgrund veränderter Rahmenbedingungen geschätzt werden. Allerdings weigert sich die Bundesregierung, daraus die Konsequenzen zu ziehen. Wenn die Politik weiterhin möchte, dass wir Zahnärzte qualitativ hochwertig arbeiten und alle Möglichkeiten der modernen Diagnose und Therapie nutzen - zum Wohle des Patienten - dann geht das auf Dauer nur mit einer angemessenen Honorierung. Darüber erst wieder im Wahljahr $2017 \mathrm{zu}$ entscheiden, klingt nach einem billigen Wahlgeschenk, mit dem die Zahnärzte milde gestimmt werden sollen. Das aber wird nicht funktionieren."

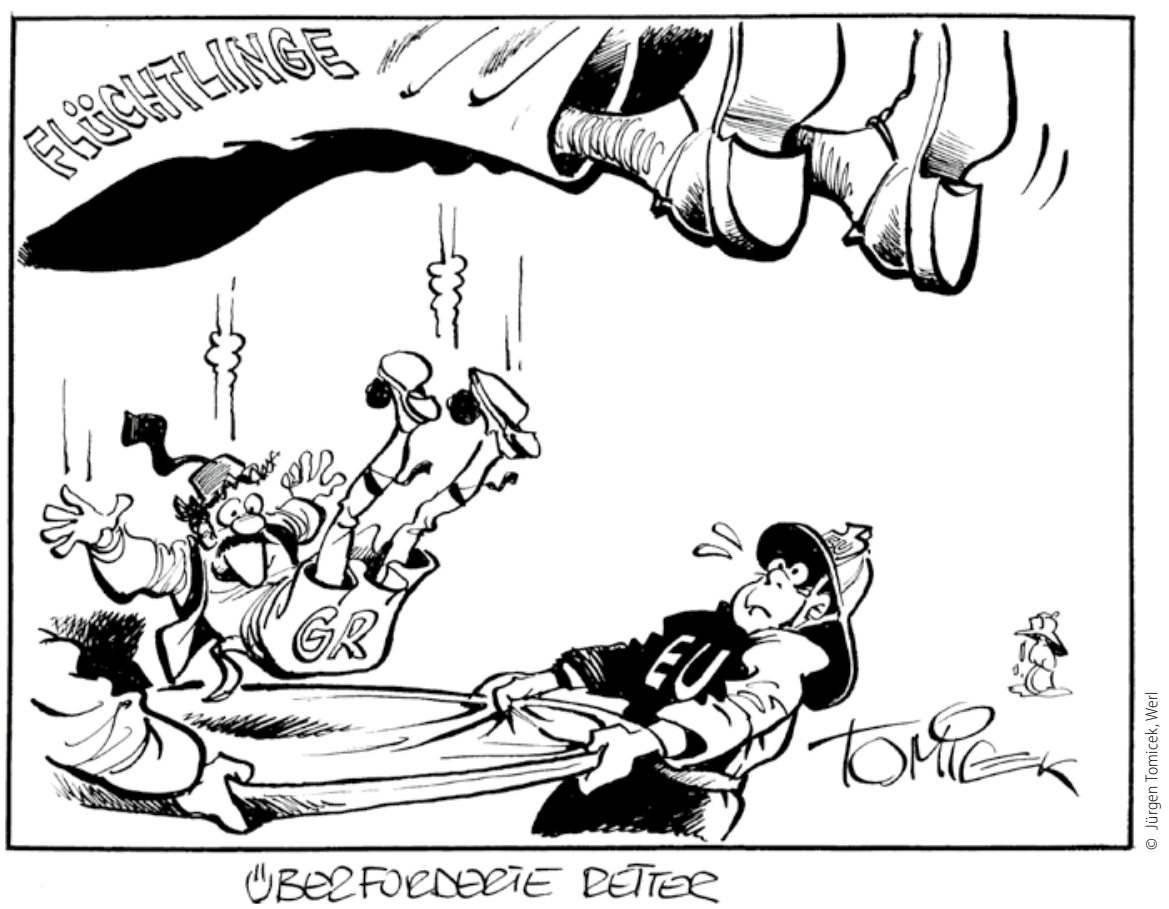

